\title{
Path Integrals on Euclidean Space Forms
}

\author{
Guillermo CAPOBIANCO and Walter REARTES
}

Departamento de Matemática, Universidad Nacional del Sur, Av. Alem 1253, 8000 Bahía Blanca, Buenos Aires, Argentina

E-mail: guillermo.capobianco@gmail.com,walter.reartes@gmail.com

Received July 09, 2015, in final form August 31, 2015; Published online September 03, 2015 http://dx.doi.org/10.3842/SIGMA.2015.071

\begin{abstract}
In this paper we develop a quantization method for flat compact manifolds based on path integrals. In this method the Hilbert space of holomorphic functions in the complexification of the manifold is used. This space is a reproducing kernel Hilbert space. A definition of the Feynman propagator, based on the reproducing property of this space, is proposed. In the $\mathbb{R}^{n}$ case the obtained results coincide with the known expressions.
\end{abstract}

Key words: path integrals; holomorphic quantization; space forms; reproducing kernel Hilbert spaces

2010 Mathematics Subject Classification: 53Z05; 81S40

\section{Introduction}

The quantization of a system whose configuration space is a differentiable manifold is a far from exhausted problem. For instance, in the case of a Riemannian manifold, it is not known which quantization scheme best represents the curvature of the manifold, see $[1,2,3,17,18,19,21$, $22,30]$. Such problems have both physical and mathematical motivations. On one side there is the problem of the existence of the structures involved in the quantization and, on the other, the possible applications to specific physical problems.

When the manifold is a compact Lie group, Hall showed that the quantization of the group is naturally isomorphic to the quantization of the cotangent space of the group, i.e., a quantization on the phase space. The latter coincides with the complexification of the group, see $[11,14,16]$. In other words, there are two Hilbert space structures, one given by the functions on the group and the other by the functions in the complexification of the group, both structures being naturally related. These problems have been explored and generalized in several other works, see, e.g., $[11,12,14,16,31]$.

It is known that the cotangent bundle of a Riemannian manifold admits a natural complex structure, compatible with the symplectic form and the natural lifting of the Riemannian metric if and only if the manifold is flat. In the Appendix A we show this result following the work of Gorbunov [6], see also [20].

In the case of an orientable connected compact flat Riemannian manifold (Euclidean space form) we show that there is a natural isomorphism between the Hilbert space of square integrable complex functions on the configuration space and the space of square integrable holomorphic functions on the phase space. The scalar products are defined with a measure given by the fundamental solution of the heat equation on each space.

This space of holomorphic functions on the phase space turns out to be a reproducing kernel Hilbert space. Taking advantage of the existence of a reproducing kernel we obtain the above mentioned isomorphism and a path integral which coincides with the known expressions in the Euclidean case, see $[5,35]$.

In particular, the 3-dimensional orientable compact Euclidean space forms present a particular interest for cosmology, since they could model the spatial part of the flat-universe models [4]. 
See the most recent works of J. Levin et al., which seek to develop a plausible cosmological model using orientable compact Euclidean space forms of dimension 3 in agreement with results of observations made on the cosmic microwave background radiation [25, 26, 27, 28].

\section{Flat Riemannian manifolds}

A connected complete flat Riemannian manifold is the quotient of $\mathbb{R}^{n}$ by a subgroup $\Gamma$ of the Euclidean group $E(n)$, which has a free and properly discontinuous action. This is a part of a more general theorem by W. Killing and H. Hopf [34].

Theorem 1. Let $M$ be a Riemannian manifold of dimension $n \geq 2$ and zero curvature. Then $M$ is complete and connected if and only if it is isometric to the quotient $\mathbb{R}^{n} / \Gamma$ with $\Gamma \subset E(n)$, where $\Gamma$ acts freely and properly discontinuously.

These manifolds are known as Euclidean space forms. The Euclidean group $E(n)$ is the semidirect product of the groups $O(n)$ and $\mathbb{R}^{n}$. An element $\gamma \in \Gamma \subset E(n)$ is identified with $\gamma=(A, a), A \in O(n)$ and $a \in \mathbb{R}^{n}$. The action of this group on an element $x \in \mathbb{R}^{n}$ is given by $\gamma(x)=A x+a$.

In one dimension the manifolds of this type are the real line $\mathbb{R}$ and the circle $S^{1}$. In dimension 2 there are five manifolds, the plane $\mathbb{R}^{2}$, the cylinder, the infinite Möebius strip, the torus and the Klein bottle. The torus and Klein bottle are both compact, while the torus is the only orientable one. In dimension 3 there are 18 types, 10 of which are compact, 6 orientable and 4 non-orientable $[23,34]$. In higher dimensions the number grows significantly; for example, there are 74 compact types in dimension 4 .

If $\Gamma \subset E(n)$ is a lattice, then the quotient $\mathbb{R}^{n} / \Gamma$ is called an $n$-torus, and is a compact Euclidean space form and a Lie group.

It can be shown that every homogeneous Riemannian manifold is diffeomorphic to some Lie group but in general a space form is not necessarily homogeneous. In particular, when the space form is homogeneous we have the following theorem [34, p. 88]:

Theorem 2. Let $M$ be a connected homogeneous Riemannian manifold of dimension $n$ and zero curvature, then it is isometric to the product $\mathbb{R}^{m} \times T^{n-m}$ of a Euclidean space with a flat Riemannian torus.

A discrete subgroup is a subgroup which is a discrete subset. If $\Gamma$ is a closed subgroup of $G$, is called uniform if the quotient space $G / \Gamma=\{g \Gamma: g \in G\}$ is compact.

The following theorem characterize discontinuous groups on Euclidean spaces.

Theorem 3. Let $\Gamma$ be a subgroup of the Euclidean group $E(n)$.

(i) $\Gamma$ acts properly discontinuously on Euclidean space $\mathbb{R}^{n}$ if, and only if, $\Gamma$ is discrete on $E(n)$.

(ii) If $\Gamma$ is closed, then it acts freely on $\mathbb{R}^{n}$ if and only it is torsion free.

(iii) $\Gamma$ acts properly discontinuously and with compact quotient on $\mathbb{R}^{n}$ if, and only if, $\Gamma$ is a discrete uniform subgroup of $E(n)$.

This paper focuses on orientable compact flat manifolds.

Flat compact Riemannian manifolds of dimension $n$ are quotients of polyhedra in $\mathbb{R}^{n}$ by identifying faces (see [34, p. 99]). The interior of these polyhedra may be taken as a chart, which we call $Q^{\circ}$. Functions defined on the manifold are functions on $\mathbb{R}^{n}$, which are invariant under the action of the group.

An important invariant for a compact Euclidean space form is its volume. This can be defined in terms of a fundamental region for $\Gamma$ in $\mathbb{R}^{n}$ [29]. The volume of a space form $\mathbb{R}^{n} / \Gamma$ is defined 
to be the volume of any fundamental region. As a fundamental region for $\Gamma$ we can take $c_{\gamma}$, the closure of the Dirichlet domain centered at $\gamma(0)$

$$
c_{\gamma}:=\left\{x \in \mathbb{R}^{n} ;\|\gamma(0)-x\| \leq\left\|\gamma^{\prime}(0)-x\right\| \text { for every } \gamma^{\prime} \in \Gamma\right\},
$$

where $\gamma(0)$ is the action of $\gamma$ on $0 \in \mathbb{R}^{n}$. $c_{\gamma}$ is an $n$-dimensional convex polyhedron in $\mathbb{R}^{n}$ bounded by hyperplanes which are perpendicular bisectors of line segments $\left[\gamma(0), \gamma^{\prime}(0)\right]$. Its boundary $\partial c_{\gamma}$ carries a locally finite decomposition into convex polyhedra of dimension $n-1$. The space form $\mathbb{R}^{n} / \Gamma$ is then obtained from $c_{\gamma}$ by identifying points in $\partial c_{\gamma}$ which are equivalent modulo $\Gamma$.

In particular, the six 3-dimensional orientable compact Euclidean space forms are the following quotient spaces $\mathbb{R}^{3} / \Gamma_{i}, i=1, \ldots, 6$ (see [34, p. 117] and [23, p. 302]). The torus $T^{3}$, which is constructed by identifying the opposite faces of a parallelepiped by translations, in this case $\Gamma_{1}$ is generated by three translations $t_{1}, t_{2}, t_{3}$, in the direction of three linear independent vectors. Other four are obtained after gluing with a quarter turn, a half-turn, a one-sixth turn and a one-third turn. The last one is the Hantzsche-Wendt space which has a more complicated structure, 3 screw motions are needed. $\Gamma_{2}$ is generated by $\Gamma_{1}$ and a screw motion $\alpha^{2}=t_{3}$, the faces of the translated parallelepiped are identified after a rotation of an angle of $\pi, \Gamma_{3}$ is generated by $\Gamma_{1}$ and a screw motion $\alpha^{3}=t_{3} . \Gamma_{4}$ is generated by $\Gamma_{1}$ and a screw motion $\alpha^{4}=t_{3}$. $\Gamma_{5}$ is generated by $\Gamma_{1}$ and a screw motion $\alpha^{6}=t_{3}$. In the last one, the Hantzsche-Wendt space, $\Gamma_{6}$ results from $\Gamma_{2}$ by adding two further screw motions by an angle of $\pi$. The manifolds $\mathbb{R}^{3} / \Gamma_{3}$ and $\mathbb{R}^{3} / \Gamma_{5}$ are obtained from a lattice made by translating a hexagonal plane lattice a certain distance perpendicular to the plane and identifying opposite sides with the top rotated by $2 \pi / 3$ and $\pi / 3$ respectively.

The family $\left\{c_{\gamma}\right\}$ forms a crystalline structure whose symmetry group contains $\Gamma$ as a subgroup of finite index (see [34, p. 100]). We can choose the interior of $c_{0}$ (the cell corresponding to the identity of the group) as the chart $Q^{\circ}$ of $\mathbb{R}^{n} / \Gamma$. The crystalline structure can be generated by translation of a finite set of vectors defining the crystal lattice. This set forms a basis of $\mathbb{R}^{n}$. Dual basis vectors multiplied by $2 \pi$ are the basis of the reciprocal lattice, $\mathcal{L}$. Let $K$ be an element of the reciprocal lattice, then a function with the symmetry of this lattice has a Fourier expansion given by

$$
f(x)=\sum_{K \in \mathcal{L}} c_{K} \mathrm{e}^{\mathrm{i} K \cdot x}
$$

This function is well defined on the manifold if it is also invariant under the action of $\Gamma$, i.e., $(\gamma f)(x)=f(\gamma x)=f(x)$ for all $\gamma \in \Gamma$.

\section{Hilbert spaces of quantization}

In a series of papers, Hall $[11,14,16]$ showed that in a compact Lie group there is a natural isomorphism between the space of square integrable functions on the group, with a measure given by the fundamental solution of the heat equation at the identity, and the space of square integrable holomorphic functions in the complexification of the group (which is isomorphic to the cotangent space). Different but equivalent versions of this isomorphism can be found in the literature, see, e.g., [11, 12, 14, 16, 31].

For orientable compact Euclidean space forms we develop this isomorphism explicitly through integrals in Euclidean space and the solutions of the heat equations. Doing so paves the way for us to write an expression for the path integral in these spaces, which is the main result of this work. 


\subsection{Weighted heat kernel representation}

First we find the fundamental solution of the heat equation (heat kernel) in a compact flat Riemannian manifold. The solution of the heat equation defined on the manifold can be calculated by finding the solution on the chart given by the polyhedron $Q^{\circ}$. Those solutions of the heat equation that are invariant under the action of the subgroup $\Gamma$ are solutions on the manifold.

If the manifold is $\mathbb{R}^{n}$, the heat equation

$$
\frac{\partial \rho_{t}^{x_{0}}(x)}{\partial t}=\frac{1}{2} \Delta \rho_{t}^{x_{0}}(x)
$$

where $\Delta$ is the Laplacian, has the following fundamental solution

$$
\rho_{t}^{x_{0}}(x)=\frac{1}{(2 \pi t)^{n / 2}} \mathrm{e}^{-\left(x-x_{0}\right)^{2} / 2 t} .
$$

This solution verifies the condition

$$
\lim _{t \rightarrow 0^{+}} \rho_{t}^{x_{0}}(x)=\delta\left(x-x_{0}\right) .
$$

In the orientable compact case, this equation can be solved on the chart $Q^{\circ}$, where we choose a point $x_{0} \in Q^{\circ}$. We seek a function $\rho_{t}^{x_{0}}(x)$ that verifies (1) with the condition (3). As was discussed above, the solution must be of the form

$$
\rho_{t}^{x_{0}}(x)=\frac{1}{V} \sum_{K \in \mathcal{L}} c_{K}(t) \mathrm{e}^{\mathrm{i} K \cdot\left(x-x_{0}\right)}
$$

where we introduced the volume $V$ of the cell because of normalization issues. Also, $\rho_{t}^{x_{0}}(x)$ must be invariant under the action of $\Gamma$. Inserting the last expression in equation (1) and considering (3), we have

$$
\rho_{t}^{x_{0}}(x)=\frac{1}{V} \sum_{K \in \mathcal{L}} \mathrm{e}^{\mathrm{i} K \cdot\left(x-x_{0}\right)-K^{2} t / 2} .
$$

This expression is invariant under the group action. It is a consequence of the symmetry of the coefficients. Indeed, if $\gamma=(A, a) \in \Gamma$, then

$$
\left(\gamma \rho_{t}\right)^{x_{0}}(x)=\frac{1}{V} \sum_{K \in \mathcal{L}} \mathrm{e}^{\mathrm{i} K \cdot \gamma\left(x-x_{0}\right)-K^{2} t / 2}=\frac{1}{V} \sum_{K \in \mathcal{L}} \mathrm{e}^{\mathrm{i} K \cdot A\left(x-x_{0}\right)+\mathrm{i} K \cdot a-K^{2} t / 2}=\rho_{t}^{x_{0}}(x) .
$$

The last equality results from the orthogonality of $A$ and $\Gamma$ having finite index in the symmetry group of the crystal. Then the fundamental solution in $Q^{\circ}$ is the fundamental solution in $Q$.

We define a scalar product on $Q$ by the following expression

$$
\langle f, g\rangle_{Q}=\int_{Q} \overline{f(x)} g(x) \rho_{t}^{x_{0}}(x) \mathrm{d} x .
$$

This product gives a Hilbert space which we call $L^{2}\left(Q, \rho_{t}^{x_{0}}\right)$. We note that this product depends on the point $x_{0}$, it is centered on a point of the manifold. The usual representation of quantum mechanics involves the space $L^{2}(Q)$ where the integration can be performed on the polyhedron $Q^{0}$ with the Lebesgue measure. The isometry between the two representations is given by

$$
f(x)=\frac{f_{S}(x)}{\sqrt{\rho_{t}^{x_{0}}(x)}}
$$

where $f_{S}$ is the corresponding function on $L^{2}(Q)$ and $f$ on $L^{2}\left(Q, \rho_{t}^{x_{0}}\right)$. Here, we name the representation on $L^{2}\left(Q, \rho_{t}^{x_{0}}\right)$ weighted heat kernel representation. 


\subsection{Holomorphic representation}

The cotangent bundle of a flat manifold has a natural complex structure. See the Appendix A.

We have seen that an appropriate chart for these manifolds is the interior of a polyhedron in $\mathbb{R}^{n}$, which we call $Q^{\circ}$. Using this chart, the cotangent bundle has a natural chart $Q^{\circ} \times \mathbb{R}^{n}$. The complex structure can be chosen so that the points of the polyhedron are the real coordinates of the complex manifold.

Any real analytic function defined on $Q^{\circ} \subset \mathbb{R}^{n}$ has an analytic extension to $Q_{\mathbb{C}}^{\circ} \subset \mathbb{C}^{n}$, where $Q_{\mathbb{C}}^{\circ}$ is $Q_{\mathbb{C}}^{\circ}=Q^{\circ} \times \mathbb{R}^{n}$. In particular, the fundamental solution (4) has an analytic extension on both variables $x$ and $x_{0}$.

Consider the heat equation on the manifold $Q_{\mathbb{C}}^{\circ}$

$$
\frac{\partial \nu_{t}^{z_{0}}(z)}{\partial t}=\frac{1}{2} \Delta \nu_{t}^{z_{0}}(z)
$$

where $z_{0}=x_{0}+\mathrm{i} y_{0}$ is a point of $Q_{\mathbb{C}}^{\circ}$ and the Laplacian is taken with respect to the $2 n$ real variables $x \in Q^{\circ}$ and $y \in \mathbb{R}^{n}$ of $z=x+\mathrm{i} y$. Given the product structure of the chart, the fundamental solution can be calculated as the product of the solution on $Q^{\circ}$ by the solution on $\mathbb{R}^{n}[7,33]$.

In the Euclidean case, the heat kernel is given by

$$
\nu_{t}^{z_{0}}(z)=\frac{1}{(2 \pi t)^{n}} \mathrm{e}^{-\left|z-z_{0}\right|^{2} / 2 t} .
$$

Furthermore, in the case of $Q_{\mathbb{C}}^{\circ}=Q^{\circ} \times \mathbb{R}^{n}$ described above we obtain

$$
\nu_{t}^{z_{0}}(z)=\frac{1}{V(2 \pi t)^{n / 2}} \mathrm{e}^{-\left|\Im\left(z-z_{0}\right)\right|^{2} / 2 t} \sum_{K \in \mathcal{L}} \mathrm{e}^{\mathrm{i} K \cdot \Re\left(z-z_{0}\right)-K^{2} t / 2} .
$$

Then we define the scalar product of holomorphic functions $\phi(z)$ and $\psi(z)$ of $Q_{\mathbb{C}}^{\circ}$ as

$$
\langle\psi, \phi\rangle_{Q_{\mathbb{C}}}=\int_{Q_{\mathbb{C}}} \overline{\psi(z)} \phi(z) \nu_{t / 2}^{x_{0}}(z) \mathrm{d} z
$$

The evaluation in $t / 2$ has been done for convenience in order to obtain the isometry (11) below.

We call $\mathcal{H} L^{2}\left(Q_{\mathbb{C}}, \nu_{t / 2}^{x_{0}}\right)$ to the Hilbert space of square integrable holomorphic functions with this scalar product. In the Euclidean case $\left(Q=\mathbb{R}^{n}\right)$ it is the Segal-Bargmann space.

The spaces of holomorphic functions shown in this paper are examples of reproducing kernel Hilbert spaces [13]. In these spaces there is a function $K(z, \bar{w})$, holomorphic in both arguments, i.e., $K$ is holomorphic in $z$ and antiholomorphic in $w$ (holomorphic in $\bar{w}$ ). For all $\phi \in \mathcal{H} L^{2}\left(Q_{\mathbb{C}}, \nu_{t / 2}^{x_{0}}\right)$ the following identity is verified

$$
\phi(z)=\int_{Q_{\mathbb{C}}} K(z, \bar{w}) \phi(w) \nu_{t / 2}^{x_{0}}(w) \mathrm{d} w .
$$

In the Euclidean case the reproducing kernel can be obtained easily [13]

$$
K(z, \bar{w})=\mathrm{e}^{z \bar{w} / t} .
$$

We consider linear operators on these spaces. Let $A$ be represented by a kernel $K_{A}(z, \bar{w})$ as follows

$$
A \phi(z)=\int_{Q_{\mathbb{C}}} K_{A}(z, \bar{w}) \phi(w) \nu_{t / 2}^{x_{0}}(w) \mathrm{d} w .
$$


Given an orthonormal basis of the Hilbert space $\left\{u_{i}(\xi)\right\}, i=1, \ldots$, the reproducing kernel can be written

$$
K(z, \bar{w})=\sum_{i=1}^{\infty} u_{i}(z) \overline{u_{i}(w)},
$$

and the corresponding kernel of the operator $A$ is given by

$$
K_{A}(z, \bar{w})=\sum_{i=1}^{\infty}\left(A u_{i}(z)\right) \overline{u_{i}(w)} .
$$

Also, the composition of operators is associated with the following kernel

$$
K_{A B}(z, \bar{w})=\int_{Q_{\mathbb{C}}} K_{A}(z, \bar{v}) K_{B}(v, \bar{w}) \nu_{t / 2}^{x_{0}}(v) \mathrm{d} v .
$$

\subsection{Isometry between Hilbert spaces}

The spaces $L^{2}\left(Q, \rho_{t}^{x_{0}}\right)$ and $\mathcal{H} L^{2}\left(Q_{\mathbb{C}}, \nu_{t / 2}^{x_{0}}\right)$ are naturally isomorphic. The isomorphism

$$
\mathcal{A}_{t}: L^{2}\left(Q, \rho_{t}^{x_{0}}\right) \rightarrow \mathcal{H} L^{2}\left(Q_{\mathbb{C}}, \nu_{t / 2}^{x_{0}}\right)
$$

is given by integration over $Q$ as follows

$$
\mathcal{A}_{t} f(z)=\int_{Q} \rho_{t}^{z}(x) f(x) \mathrm{d} x
$$

where $\rho_{t}^{z}(x)$ is the analytic extension of $\rho_{t}^{x_{0}}(x)$ in the variable $x_{0}$.

The last expression is an isomorphism that can be explicitly tested as follows using the expressions for the kernels of the heat equations

$$
\begin{aligned}
\left\langle\mathcal{A}_{t} f, \mathcal{A}_{t} g\right\rangle_{Q_{\mathbb{C}}} & =\int_{Q_{\mathbb{C}}} \overline{\mathcal{A}_{t} f(z)} \mathcal{A}_{t} g(z) \nu_{t / 2}^{x_{0}}(z) \mathrm{d} z \\
& =\int_{Q \times Q} \overline{f(x)} g\left(x^{\prime}\right) \int_{Q_{\mathbb{C}}} \overline{\rho_{t}^{z}(x)} \rho_{t}^{z}\left(x^{\prime}\right) \nu_{t / 2}^{x_{0}}(z) \mathrm{d} z \mathrm{~d} x \mathrm{~d} x^{\prime} \\
& =\int_{Q \times Q} \overline{f(x)} g\left(x^{\prime}\right) \rho_{t}^{x_{0}}\left(x^{\prime}\right) \delta\left(x-x^{\prime}\right) \mathrm{d} x \mathrm{~d} x^{\prime}=\langle f, g\rangle_{Q} .
\end{aligned}
$$

The integral over $Q_{\mathbb{C}}$ in the second line is evaluated using the expressions (5) and (7) along with the usual orthogonality relations.

This form of the isometry is the analogous to what Hall calls $B_{t}$ [11]. There are other forms, however we continue using (12) for the purpose of finding the path integral.

\subsection{The $S^{1}$ case}

Now we consider the case in which $Q=S^{1}$. The fundamental solution $\rho_{t}^{\theta_{0}}(\theta)$, centered on $\theta_{0}$, satisfies the heat equation

$$
\frac{\partial \rho_{t}^{\theta_{0}}(\theta)}{\partial t}=\frac{1}{2} \Delta_{S^{1}} \rho_{t}^{\theta_{0}}(\theta)
$$

and converges to the Dirac delta $\delta\left(\theta-\theta_{0}\right)$, for $t \rightarrow 0^{+}$. 
The function $\rho_{t}^{\theta_{0}}$ is given by

$$
\rho_{t}^{\theta_{0}}(\theta)=\sum_{k=-\infty}^{\infty} \rho_{k}(t) \mathrm{e}^{\mathrm{i} k\left(\theta-\theta_{0}\right)},
$$

and therefore the functions $\rho_{k}$ satisfy

$$
\frac{\mathrm{d} \rho_{k}(t)}{\mathrm{d} t}=-\frac{1}{2} k^{2} \rho_{k}(t),
$$

and we finally obtain

$$
\rho_{t}^{\theta_{0}}(\theta)=\frac{1}{2 \pi} \sum_{k \in \mathbb{Z}} \mathrm{e}^{\mathrm{i} k\left(\theta-\theta_{0}\right)-\frac{1}{2} k^{2} t} .
$$

Then the scalar product of two functions $f$ and $g$ on $S^{1}$ is

$$
\begin{aligned}
\langle f, g\rangle & =\frac{1}{2 \pi} \int_{-\pi}^{\pi} \sum_{m, n=-\infty} \bar{c}_{m} d_{n} \mathrm{e}^{\mathrm{i} \theta(n-m)} \sum_{k=-\infty}^{\infty} \mathrm{e}^{\mathrm{i} k\left(\theta-\theta_{0}\right)-k^{2} t / 2} \mathrm{~d} \theta \\
& =\sum_{m, n=-\infty}^{\infty} \bar{c}_{m} d_{n} \mathrm{e}^{-\mathrm{i}(m-n) \theta_{0}-(m-n)^{2} t / 2}=\sum_{k=-\infty}^{\infty}\left(\sum_{j=-\infty}^{\infty} \bar{c}_{j} d_{j-k}\right) \mathrm{e}^{-\mathrm{i} k \theta_{0}-k^{2} t / 2},
\end{aligned}
$$

where $c_{j}$ and $d_{j}$ are the Fourier coefficients of $f$ and $g$, respectively.

The cotangent space is the cylinder $S^{1} \times \mathbb{R}$. Thus, the complex manifold $S_{\mathbb{C}}^{1}$ can be represented by the chart $(-\pi, \pi) \times \mathbb{R}$ viewed as the vertical strip in the complex plane.

Holomorphic functions defined on the cylinder are holomorphic functions on $\mathbb{C}$, which are also periodic on the coordinate corresponding to the real part. Using the Cauchy-Riemann conditions it is straightforward to see that they are of the form

$$
\psi(w)=\sum_{l=-\infty}^{\infty} \psi_{l} \mathrm{e}^{\mathrm{i} l w} .
$$

Given that the heat kernel on a product manifold is the product of the respective heat kernels [7, 8], in this case in particular the heat kernel on $S^{1} \times \mathbb{R}$ is obtained from the heat kernel on $S^{1}(13)$ and the heat kernel on $\mathbb{R}(2)$, respectively.

Then, the measure $\nu_{t}^{z_{0}}(z)$ in this case is

$$
\nu_{t}^{z_{0}}(z)=\frac{1}{2 \pi \sqrt{2 \pi t}} \mathrm{e}^{-\frac{\Im\left(z-z_{0}\right)^{2}}{2 t}} \sum_{n=-\infty}^{\infty} \mathrm{e}^{\mathrm{i} n \Re\left(z-z_{0}\right)-n^{2} t / 2} .
$$

The Segal-Bargmann transform (12) of a function $f$ can be obtained easily from his Fourier coefficients. It is given by

$$
\psi(z)=\frac{1}{2 \pi} \int_{-\pi}^{\pi} f(\theta) \sum_{n=-\infty}^{\infty} \mathrm{e}^{\mathrm{i} n(\theta-z)-n^{2} t / 2} \mathrm{~d} \theta=\sum_{m=-\infty}^{\infty} c_{m} \mathrm{e}^{\mathrm{i} m z-m^{2} t / 2} .
$$

Given that $\left\{\mathrm{e}^{\mathrm{i} n x} / \sqrt{2 \pi}\right\}$ with integer $n$ is an orthonormal basis of $L^{2}\left(S^{1}\right)$, using (6) and (12) we can obtain $\left\{\phi_{n}\right\}$, which is an orthonormal basis of $\mathcal{H} L^{2}\left(S^{1}, \nu_{t}^{x_{0}}\right)$,

$$
\phi_{n}(z)=\frac{1}{\sqrt{2 \pi}} \int_{-\pi}^{\pi} \frac{\mathrm{e}^{\mathrm{i} n x} \rho_{t}^{z}(x)}{\sqrt{\rho_{t}^{x_{0}}(x)}} \mathrm{d} x .
$$

Then, by equation (10) the reproducing kernel is

$$
K(z, \bar{w})=\frac{1}{2 \pi} \sum_{k \in \mathbb{Z}} \int_{-\pi}^{\pi} \int_{-\pi}^{\pi} \frac{\mathrm{e}^{\mathrm{i} k x} \mathrm{e}^{-\mathrm{i} k y}}{\sqrt{\rho_{t}^{x_{0}}(x) \rho_{t}^{x_{0}}(y)}} \rho_{t}^{z}(x) \rho_{t}^{\bar{w}}(y) \mathrm{d} x \mathrm{~d} y=\frac{1}{2 \pi} \int_{-\pi}^{\pi} \frac{\rho_{t}^{z}(x) \rho_{t}^{\bar{w}}(x)}{\rho_{t}^{x_{0}}(x)} \mathrm{d} x .
$$




\section{The Feynman path integral}

In this section we propose a definition of the Feynman integral in the holomorphic representation which is suitable for flat manifolds. Here, we take a heuristic motivation for our definition.

First we consider the propagation of the wave function $\phi$ for a small time $\epsilon$. The propagation is governed by the Schrödinger equation with Hamiltonian operator $H$. We call $K_{H}$ to the kernel of this Hamiltonian in the holomorphic representation.

We obtain the evolution operator by exponentiating the Hamiltonian. Applying the evolution operator to (8), using (9) and introducing the series expansion of the exponential we obtain

$$
\begin{aligned}
\mathrm{e}^{-\mathrm{i} \epsilon H} \phi(z) & =\int_{Q_{\mathbb{C}}}\left(\sum_{n=0}^{\infty} \frac{(-\mathrm{i} \epsilon)^{n}}{n !} K_{H^{n}}(z, \bar{w})\right) \phi(w) \nu_{t / 2}^{x_{0}}(w) \mathrm{d} w \\
& =\int_{Q_{\mathbb{C}}} K(z, \bar{w}) \mathrm{e}^{-\mathrm{i} \epsilon K_{H}(z, \bar{w}) / K(z, \bar{w})} \phi(w) \nu_{t / 2}^{x_{0}}(w) \mathrm{d} w+\epsilon^{2} \psi(z, \epsilon)
\end{aligned}
$$

Thus, calling $U_{\epsilon} \phi(z)=\mathrm{e}^{-\mathrm{i} \epsilon H} \phi(z)$ and $\tilde{U}_{\epsilon} \phi(z)$ to the last integral, we have

$$
U_{\epsilon} \phi(z)=\tilde{U}_{\epsilon} \phi(z)+\epsilon^{2} \psi(z, \epsilon) .
$$

If we divide a time interval $T$ into $n$ equal parts, i.e., $T=n \epsilon$, we have

$$
U_{T}=\lim _{n \rightarrow \infty} U_{T / n}^{n}
$$

then

$$
U_{T} \phi(z)=\lim _{n \rightarrow \infty} U_{T / n}^{n} \phi(z)=\lim _{n \rightarrow \infty}\left(\tilde{U}_{T / n}^{n} \phi(z)+\left(\frac{T}{n}\right)^{2} \Gamma(z, T / n)\right)=\tilde{U}_{T} \phi(z) .
$$

We define a Feynman propagator for a finite time $T$ by dividing the interval $[0, T]$ into $n$ equal subintervals and taking the limit $n \rightarrow \infty$. Then, it follows

$$
G\left(z_{T}, z_{0} ; T\right)=\lim _{n \rightarrow \infty} \int_{Q_{\mathbb{C}}^{n-1}} \mathrm{e}^{-\mathrm{i} \frac{T}{n} \sum_{j=1}^{n} h\left(z_{j}, \bar{z}_{j-1}\right)} \prod_{j=1}^{n} K\left(z_{j}, \bar{z}_{j-1}\right) \prod_{j=1}^{n-1} \nu_{t / 2}^{x_{0}}\left(z_{j}\right) \mathrm{d} z_{j},
$$

where we introduce the normal symbol of the Hamiltonian

$$
h(z, \bar{w})=\frac{K_{H}(z, \bar{w})}{K(z, \bar{w})} .
$$

The above expression can also be calculated in the Euclidean case. Here, we take the Hamiltonian

$$
H=z \frac{\partial}{\partial z},
$$

corresponding to a renormalized one-dimensional harmonic oscillator. The kernel of this Hamiltonian is $K_{H}(z, \bar{w})=z \bar{w} \mathrm{e}^{z \bar{w}}$ and the normal symbol $h(z, \bar{w})=z \bar{w}$.

The Feynman propagator (14) in this case is

$$
G\left(z_{T}, z_{0} ; T\right)=\lim _{n \rightarrow \infty} \int_{\mathbb{C}^{n-1}} \mathrm{e}^{\sum_{j=1}^{n} z_{j} \bar{z}_{j-1}-\mathrm{i} \frac{T}{n} \sum_{j=1}^{n} z_{j} \bar{z}_{j-1}-\sum_{j=1}^{n-1}\left|z_{j}\right|^{2}} \prod_{j=1}^{n-1} \frac{\mathrm{d} z_{j}}{2 \pi} .
$$


Then, by regrouping terms, we have

$$
G\left(z_{T}, z_{0} ; T\right)=\lim _{n \rightarrow \infty} \int_{\mathbb{C}^{n-1}} \mathrm{e}^{z_{n} \bar{z}_{n-1}-\epsilon \sum_{j=1}^{n-1} z_{j} \frac{\left(\bar{z}_{j}-\bar{z}_{j-1}\right)}{\epsilon}-\mathrm{i} \epsilon \sum_{j=1}^{n-1} z_{j} \bar{z}_{j-1}-\mathrm{i} \epsilon z_{n} \bar{z}_{n-1}} \prod_{j=1}^{n-1} \frac{\mathrm{d} z_{j}}{2 \pi}
$$

where again $\epsilon=T / n$.

This expression is the known Feynman integral

$$
G\left(z_{T}, z_{0} ; T\right)=\int \mathrm{e}^{z(T) \bar{z}(T)} \mathrm{e}^{\mathrm{i} \int_{0}^{T}(\mathrm{i} z(s) \dot{\bar{z}}(s)-h(z(s), \bar{z}(s))) \mathrm{d} s} \mathcal{D}[z(s)]
$$

shown in $[5,35]$.

\section{Discussion}

The cotangent bundle of a Riemannian manifold admits a complex structure compatible with the symplectic form an the natural lifting of the Riemannian metric if and only if the manifold is flat, i.e., this structure is not integrable unless the curvature is zero, see Appendix A.

The restriction on the integrability of the almost-complex structure can be circumvented by changing the metric. In [6], in the context of deformation quantization, Gorbunov et al. constructed a formally Kähler metric on the cotangent space by adding powers of momentum, obtaining an integrable Kähler structure.

Furthermore, it has been shown that a complex manifold can be constructed in a neighborhood of the null section of the tangent bundle of a real Riemannian manifold. These are called Grauert tubes [9, 10, 24, 32]. The complex structures defined therein, called adapted complex structures, are compatible with the symplectic structure leading to a Kähler manifold [15]. In certain cases this complex structure exists throughout the tangent, for example when the base manifold is a compact Lie group with a bi-invariant metric.

Our paper is focused on flat Riemannian manifolds, specifically on Euclidean space forms. They present a particular interest in cosmology because they could model the spatial part of flat universe models, see $[4,25]$. The formulas presented in this paper are applicable only to flat manifolds. However the method that leads to the formulation of the path integral is interesting in itself. Moreover, it allows exploring the quantization of space-forms, a topic sparsely discussed in the literature. Finally, the method presented here could be useful for generalizations in future works.

\section{A Complex structure in phase space}

It is known that the cotangent bundle of a Riemannian manifold admits a natural complex structure, compatible with the symplectic form and the natural lifting of the Riemannian metric if and only if the manifold is flat $[6,20]$.

First we consider the general case. Let $Q$ be a finite-dimensional orientable real-analytic Riemannian manifold and let $T^{*} Q$ be the cotangent bundle with projection $\pi: T^{*} Q \rightarrow Q$ and canonical symplectic form $\omega$.

The metric in $Q$ can be naturally lifted to the cotangent space $T^{*} Q$ as follows. Let $\alpha(t)=$ $\left(q_{1}(t), p_{1}(t)\right)$ and $\beta(t)=\left(q_{2}(t), p_{2}(t)\right)$ be curves on $T^{*} Q$ such that

$$
\alpha(0)=\beta(0)=(q, p)=m, \quad \alpha^{\prime}(0)=V, \quad \text { and } \quad \beta^{\prime}(0)=W,
$$


i.e., $V$ and $W$ are tangent vectors on $m$. We denote by $\sigma$ the metric on $Q$, and by $\sigma^{\sharp}$ the isomorphism induced by $\sigma$ between $T^{*} Q$ and $T Q$. Then we have a metric $G$ on $T^{*} Q$ given by the following expression

$$
G_{m}(V, W)=\sigma_{q}(T \pi V, T \pi W)+\sigma_{q}\left(\sigma_{q}^{\sharp} \frac{D p_{1}}{\mathrm{~d} t}(0), \sigma_{q}^{\sharp} \frac{D p_{2}}{\mathrm{~d} t}(0)\right) .
$$

Here, $T \pi$ is the tangent application of the projection and $D$ represents the covariant derivative.

Thus, we have two different structures on $T^{*} Q$; the canonical symplectic structure $\omega$ and the Riemannian metric $G$. A third one appears naturally. It is the almost-complex structure $J$. If $\omega^{\sharp}: T^{*} T^{*} Q \rightarrow T T^{*} Q$ is the isomorphism induced by $\omega$, and $G^{b}: T T^{*} Q \rightarrow T^{*} T^{*} Q$ the isomorphism induced by $G$, then $J$ is given by $J=\omega^{\sharp} G^{b}$. It means that given the fields $V$ and $W$, they verify

$$
G(V, W)=\omega(V, J W) .
$$

At each point $m$ the map $J_{m}: T_{m} T^{*} Q \rightarrow T_{m} T^{*} Q$ verifies $J_{m}^{2} V=-V . J$ is compatible with $\omega$ and $G$. $(J, G, \omega)$ form what is known as a compatible triple.

We are now interested in finding appropriate local complex coordinates. The complexified tangent bundle $T^{\mathbb{C}} T^{*} Q$ splits as follows

$$
T^{\mathbb{C}} T^{*} Q=T^{(1,0)} T^{*} Q \oplus T^{(0,1)} T^{*} Q .
$$

Here $T^{(1,0)} T^{*} Q$ and $T^{(0,1)} T^{*} Q$ are the images of the projections $\Pi^{+}$and $\Pi^{-}$given by

$$
\Pi^{ \pm}=\frac{1 \mp \mathrm{i} J}{2}
$$

If we choose a vector $V=\left(\dot{q}^{1}, \ldots, \dot{q}^{n}, \dot{p}_{1}, \ldots, \dot{p}_{n}\right) \in T_{m} T^{*} Q$, then $\Pi^{+}$is a natural isomorphism between the real tangent space $T_{m} T^{*} Q$ and the holomorphic tangent space $T_{m}^{(1,0)} T^{*} Q$. Explicitly, we have

$$
\Pi^{+} V=\dot{z}^{i} \frac{\partial}{\partial z^{i}},
$$

where $\dot{z}^{i}=\dot{q}^{i}+\mathrm{i} \sigma^{i m}\left(\dot{p}_{m}-p_{k} \Gamma_{m l}^{k} \dot{q}^{l}\right)$.

The corresponding holomorphic vector fields are

$$
\frac{\partial}{\partial z^{i}}=\frac{1}{2}\left(\frac{\partial}{\partial q^{i}}+p_{k} \Gamma_{i j}^{k} \frac{\partial}{\partial p^{j}}-\mathrm{i} \sigma_{i j} \frac{\partial}{\partial p^{j}}\right),
$$

where $\sigma_{i j}$ and $\Gamma_{i j}^{k}$ are the matrix coefficients of the metric and the Christoffel symbols respectively. Henceforth, the Einstein summation convention is used.

If we take the Lie bracket of the above-mentioned fields we obtain

$$
\left[\frac{\partial}{\partial z^{i}}, \frac{\partial}{\partial z^{j}}\right]=\mathrm{i} R_{k i j}^{m} p_{m} \sigma^{l k}\left(\frac{\partial}{\partial z^{l}}-\frac{\partial}{\partial \bar{z}^{l}}\right) \text {. }
$$

Then, by the Niremberg-Newlander theorem [20], the distribution is integrable if and only if the curvature tensor of the metric $\sigma$ is identically null, see Gorbunov et al. [6].

\section{Acknowledgements}

We thank Hernán Cendra for his reading of the manuscript and useful suggestions. This work was supported by the Universidad Nacional del Sur (Grants PGI 24/L085, PGI 24/L086 and PGI 24/ZL10). 


\section{References}

[1] Casimir H.B.G., Rotation of a rigid body in quantum mechanics, Ph.D. Thesis, Rijksuniversiteit te Leiden, 1931.

[2] Chaichian M., Demichev A., Path integrals in physics. Vol. I. Stochastic processes and quantum mechanics, Series in Mathematical and Computational Physics, Institute of Physics Publishing, Bristol, 2001.

[3] DeWitt B.S., Dynamical theory in curved spaces. I. A review of the classical and quantum action principles, Rev. Mod. Phys. 29 (1957), 377-397.

[4] Ellis G.F.R., Topology and cosmology, Gen. Relativity Gravitation 2 (1971), 7-21.

[5] Faddeev L., Elementary introduction to quantum field theory, in Quantum Fields and Strings: a Course for Mathematicians, Vols. 1, 2 (Princeton, NJ, 1996/1997), Editors P. Deligne, P. Etingof, D.S. Fred, L.C. Jeffrey, D. Kazhdan, J.W. Morgan, D.R. Morrison, E. Witten, Amer. Math. Soc., Providence, RI, 1999, 513-550.

[6] Gorbunov I.V., Lyakhovich S.L., Sharapov A.A., Wick quantization of cotangent bundles over Riemannian manifolds, J. Geom. Phys. 53 (2005), 98-121, hep-th/0401022.

[7] Grigor'yan A., Heat kernels on weighted manifolds and applications, in The Ubiquitous Heat Kernel, Contemp. Math., Vol. 398, Editors J. Jorgenson, L. Walling, Amer. Math. Soc., Providence, RI, 2006, 93-191.

[8] Grigor'yan A., Heat kernel and analysis on manifolds, AMS Studies in Advanced Mathematics, Vol. 47, Amer. Math. Soc., Providence, RI, 2009.

[9] Guillemin V., Stenzel M., Grauert tubes and the homogeneous Monge-Ampère equation, J. Differential Geom. 34 (1991), 561-570.

[10] Guillemin V., Stenzel M., Grauert tubes and the homogeneous Monge-Ampère equation. II, J. Differential Geom. 35 (1992), 627-641.

[11] Hall B.C., The Segal-Bargmann "coherent state" transform for compact Lie groups, J. Funct. Anal. 122 (1994), 103-151.

[12] Hall B.C., The inverse Segal-Bargmann transform for compact Lie groups, J. Funct. Anal. 143 (1997), 98-116.

[13] Hall B.C., Holomorphic methods in analysis and mathematical physics, in First Summer School in Analysis and Mathematical Physics (Cuernavaca Morelos, 1998), Contemp. Math., Vol. 260, Editors S. Pérez-Esteva, C. Vilegas-Blas, Amer. Math. Soc., Providence, RI, 2000, 1-59, quant-ph/9912054.

[14] Hall B.C., Harmonic analysis with respect to heat kernel measure, Bull. Amer. Math. Soc. 38 (2001), 43-78, quant-ph/0006037.

[15] Hall B.C., Kirwin W.D., Adapted complex structures and the geodesic flow, Math. Ann. 350 (2011), 455474, arXiv:0811.3083.

[16] Hall B.C., Mitchell J.J., The Segal-Bargmann transform for noncompact symmetric spaces of the complex type, J. Funct. Anal. 227 (2005), 338-371, quant-ph/0409118.

[17] Kleinert H., Quantum mechanics and path integrals in spaces with curvature and torsion, Modern Phys. Lett. A 4 (1989), 2329-2337.

[18] Kleinert H., Path integral on spherical surfaces in $D$ dimensions and on group spaces, Phys. Lett. B 236 (1990), 315-320.

[19] Kleinert H., Path integrals in quantum mechanics, statistics, polymer physics, and financial markets, 3rd ed., World Sci. Publ. Co., Inc., River Edge, NJ, 2004.

[20] Kobayashi S., Nomizu K., Foundations of differential geometry. Vol. II, Interscience Tracts in Pure and Applied Mathematics, Vol. 15, John Wiley \& Sons, Inc., New York - London - Sydney, 1969.

[21] Kowalski K., Rembieliński J., Quantum mechanics on a sphere and coherent states, J. Phys. A: Math. Gen. 33 (2000), 6035-6048.

[22] Kowalski K., Rembieliński J., Papaloucas L.C., Coherent states for a quantum particle on a circle, J. Phys. A: Math. Gen. 29 (1996), 4149-4167, quant-ph/9801029.

[23] Kühnel W., Differential geometry. Curves - surfaces - manifolds, Student Mathematical Library, Vol. 16, Amer. Math. Soc., Providence, RI, 2002.

[24] Lempert L., Szőke R., Global solutions of the homogeneous complex Monge-Ampère equation and complex structures on the tangent bundle of Riemannian manifolds, Math. Ann. 290 (1991), 689-712. 
[25] Levin J., Topology and the cosmic microwave background, Phys. Rep. 365 (2002), 251-333, gr-qc/0108043.

[26] Levin J., Scannapieco E., de Gasperis G., Silk J., Barrow J.D., How the universe got its spots, Phys. Rev. D 58 (1998), 123006, 14 pages, astro-ph/9807206.

[27] Levin J., Scannapieco E., Silk J., Is the universe infinite or is it just really big?, Phys. Rev. D 58 (1998), 103516, 5 pages, astro-ph/9802021.

[28] Levin J., Scannapieco E., Silk J., The topology of the universe: the biggest manifold of them all, Classical Quantum Gravity 15 (1998), 2689-2697, gr-qc/9803026.

[29] McMullen P., Schulte E., Abstract regular polytopes, Encyclopedia of Mathematics and its Applications, Vol. 92, Cambridge University Press, Cambridge, 2002.

[30] Mostafazadeh A., Scalar curvature factor in the Schrödinger equation and scattering on a curved surface, Phys. Rev. A 54 (1996), 1165-1170, hep-th/9602095.

[31] Stenzel M.B., The Segal-Bargmann transform on a symmetric space of compact type, J. Funct. Anal. 165 (1999), 44-58.

[32] Szőke R., Complex structures on tangent bundles of Riemannian manifolds, Math. Ann. 291 (1991), 409-428.

[33] van Leeuwen S., The Segal-Bargmann transform and its generalizations, Master's thesis, Universiteit Utrecht, 2009.

[34] Wolf J.A., Spaces of constant curvature, 6th ed., AMS Chelsea Publishing, Providence, RI, 2011.

[35] Zinn-Justin J., Path integrals in quantum mechanics, Oxford Graduate Texts, Oxford University Press, Oxford, 2005. 